\title{
New Soft Theorems for Goldstone-Boson Amplitudes
}

\author{
Karol Kampfø, ${ }^{1}$ Jiri Novotny $\odot,{ }^{1}$ Mikhail Shifman, ${ }^{2}$ and Jaroslav Trnka ${ }^{3,1}$ \\ ${ }^{1}$ Institute of Particle and Nuclear Physics, Charles University, Prague 18000, Czech Republic \\ ${ }^{2}$ William I. Fine Theoretical Physics Institute, University of Minnesota, Minneapolis, Minnesota 55455, USA \\ ${ }^{3}$ Center for Quantum Mathematics and Physics (QMAP), University of California, Davis, California 95616, USA
}

(Received 12 November 2019; accepted 14 February 2020; published 18 March 2020)

\begin{abstract}
In this Letter we discuss new soft theorems for the Goldstone-boson amplitudes with nonvanishing soft limits. The standard argument is that the nonlinearly realized shift symmetry leads to the vanishing of scattering amplitudes in the soft limit, known as the Adler zero. This statement involves certain assumptions of the absence of cubic vertices and the absence of linear terms in the transformations of fields. For theories which fail to satisfy these conditions, we derive a new soft theorem which involves certain linear combinations of lower point amplitudes, generalizing the Adler zero statement. We provide an explicit example of the $\mathrm{SU}(N) / \mathrm{SU}(N-1)$ sigma model which was also recently studied in the context of U(1) fibrated models. The soft theorem can be then used as an input into the modified soft recursion relations for the reconstruction of all tree-level amplitudes.
\end{abstract}

DOI: 10.1103/PhysRevLett.124.111601

Introduction.-In this Letter we connect two different topics that have been intensively studied in the past few years: soft limits of scattering amplitudes in effective field theories and the $\mathrm{U}(1)$ fibrated $C P(N-1)$ sigma models. The tree-level $S$ matrix in these models exhibits a very special behavior in the soft limit which gives rise to the new type of soft theorems, distinct from the usual Adler zero.

Sigma models. - The U(1) fibrated $C P(N-1)$ models represent a class of sigma models interpolating between $C P(N-1)$ and $S^{2 N-1}$ target spaces [1-3]. These models correspond to the cosets $[(\mathrm{SU}(N) / \mathrm{SU}(N-1) \times \mathrm{U}(1))] \times$ $\mathrm{U}(1)$. For brevity, in the following we refer to these models as $\mathrm{SU}(N) / \mathrm{SU}(N-1)$. The above class contains an extremely interesting example of $N=2$, including $\mathrm{CP}(1)$ and $S^{3}$ models, both being integrable and exactly solvable in two spacetime dimensions [3-6]. The algebraic form of the interpolating Lagrangian is

$$
\mathcal{L}=\frac{1}{2 \lambda^{2}}\left\{\left[\sum_{a=1,2,3} J_{\mu}^{a} J^{a \mu}\right]-\kappa J_{\mu}^{3} J^{3 \mu}\right\},
$$

where the current $J_{\mu}$ is defined as

$J_{\mu}=U^{\dagger} \partial_{\mu} U \equiv 2 i \sum J_{\mu}^{a} T^{a}, \quad J_{\mu}^{a}=-i \operatorname{Tr}\left(J_{\mu} T^{a}\right)$.

Here $U$ is an arbitrary $x$-dependent matrix, $\mathrm{U}(x) \in \mathrm{SU}(2)$, the generators are proportional to the Pauli matrices,

Published by the American Physical Society under the terms of the Creative Commons Attribution 4.0 International license. Further distribution of this work must maintain attribution to the author(s) and the published article's title, journal citation, and DOI. Funded by SCOAP ${ }^{3}$.
$T^{a}=\frac{1}{2} \tau^{a}$, and $\kappa$ is a numerical deformation parameter. If $\kappa=1$, the theory is equivalent to the $\mathrm{CP}(1)$ model, while at $\kappa=0$ it reduces to the $\mathrm{SU}(2) \times \mathrm{SU}(2) / \mathrm{SU}(2)$ principal chiral model (PCM) whose target space is $S^{3}$. For arbitrary $N$ we can extend Eq. (1) as follows:

$$
\mathcal{L}=\frac{1}{2 \lambda^{2}} \sum_{a=1}^{2 N-2}\left[\left(J^{N^{2}-2 N+a}\right)^{2}+\frac{1-\kappa}{N}\left(J^{N^{2}-1}\right)^{2}\right] .
$$

Scattering amplitudes.-Recently, there has been huge progress in new methods for the calculation of on-shell scattering amplitudes in quantum field theory (QFT). While most work has been focused on gauge theory and gravity, especially with maximal supersymmetry, new surprising results have been obtained in the case of effective field theories (EFTs). The general approach is to fix the amplitude uniquely by imposing certain sets of constraints. The universal example is a tree-level factorization on poles,

$$
\lim _{P^{2} \rightarrow 0} A_{n}=\sum \frac{A_{L} A_{R}}{P^{2}}
$$

where the sum runs over internal states. The set of all factorizations is enough to completely specify the tree-level $S$ matrix in a large class of QFTs, called on-shell constructible, including gauge theories or gravity, and it can then be calculated using the recursion relations [7].

This does not apply to EFTs due to the presence of unfixed contact terms with no poles, which originate from higher-dimensional operators in the Lagrangian. In Ref. [8] it was shown that when the amplitude vanishes for one of the momenta going to zero, we can impose this information 
as a constraint and use soft recursion relations for on-shell reconstruction. This singles out a set of exceptional EFTs where all coefficients in the Lagrangian are fixed by the requirement of a certain degree of vanishing, $A_{n}=\mathcal{O}\left(p^{\sigma}\right)$, in the soft limit [8-14].

The primary example is the PCM describing the spontaneous symmetry breaking $\mathrm{SU}(N) \times \mathrm{SU}(N) \rightarrow \mathrm{SU}(N)$. It has been known since 1970 [15] that the requirement of the vanishing soft limit of amplitudes, known also as the Adler zero, on any two-derivate theory specifies nonlinear sigma model (NLSM) as a unique solution. In Ref. [16] it was found that in this model the group part of tree-level amplitudes can be stripped, similar to Yang-Mills amplitudes, dramatically simplifying the calculations.

Adler zero.-First we review the standard textbook derivation of the Adler zero for amplitudes of NambuGoldstone bosons (NGBs). We start with the theory for the single NGB corresponding to the spontaneous breaking of one-parameter continuous symmetry. The NGB couples to the associated Noether current $N^{\mu}(x)$ with a strength parametrized by the decay constant $F$ :

$$
\left\langle 0\left|N^{\mu}(x)\right| \phi(p)\right\rangle=-i p^{\mu} F e^{-i p \cdot x} .
$$

The matrix element of this current between physical states has a pole for $p^{2} \rightarrow 0$, and the residue corresponds to the scattering amplitude for the NGB emission. For the element between out state $\langle\alpha|$ and in state $|\beta\rangle$, we get

$$
\left\langle\alpha\left|N^{\mu}(0)\right| \beta\right\rangle=F \frac{p^{\mu}}{p^{2}} A_{n}(\alpha+\phi(p), \beta)+R^{\mu}(p) .
$$

Here $A_{n}(\alpha+\phi(p), \beta)$ is the on-shell amplitude which involves emission of the state $\phi$ with momentum $p$, where $p^{\mu}=P_{\beta}^{\mu}-P_{\alpha}^{\mu}$ is the difference between incoming and outgoing momenta, and $R^{\mu}(p)$ is the regular function for $p^{2} \rightarrow 0$. Because of the conservation of the current, we have $p_{\mu}\left\langle\alpha\left|N^{\mu}(0)\right| \beta\right\rangle=0$ and, therefore,

$$
A_{n}(\alpha+\phi(p), \beta)=-\frac{1}{F} p_{\mu} R^{\mu}(p) .
$$

Suppose that $R^{\mu}(p)$ is regular also in the limit $p \rightarrow 0$. This is an additional assumption which does not follow automatically from the standard polology. Then the amplitude $A_{n}$ vanishes if the NGB momentum is soft,

$$
\lim _{p \rightarrow 0} A_{n}(\alpha+\phi(p), \beta)=0 .
$$

This is the statement of the Adler zero. The same argument applies to the theory with multiple Goldstone bosons. To summarize, we have the nonperturbative Adler zero provided the matrix element $\left\langle\alpha\left|N^{\mu}(0)\right| \beta\right\rangle$ of the Noether current corresponding to the spontaneous symmetry breaking has no other singularity for $p \rightarrow 0$ besides the NGB pole. Therefore, the violation of the Adler zero is possible only when there are additional singularities in the matrix element of the Noether current. This is achieved in the case when the Noether current can be inserted into the external lines of the amplitude $A_{n}(\alpha, \beta)$, i.e., when there are quadratic terms in the expansion of the operator $N^{\mu}$ in the elementary fields. There are two sources of these quadratic terms: (1) the presence of cubic vertices in the Lagrangian and (2) the presence of linear terms in the nonlinearly realized symmetry transformation corresponding to the Noether current $N^{\mu}$. Schematically,

$$
\delta \phi=a+b \phi+\mathcal{O}\left(\phi^{2}\right), \quad b \neq 0 .
$$

These two conditions are not sufficient: even when at least one of the above conditions is satisfied, the theory can still have the Adler zero-a more detailed analysis is needed. Note that the cubic vertices can always be removed by means of field redefinitions, as there are no on-shell three-point amplitudes (apart from $\phi^{3}$ theory). In such a case the presence of the linear term in $\delta \phi$ is crucial. Note that, e.g., in the PCM parametrized by the Lagrangian,

$\mathcal{L}=F^{2} \operatorname{Tr}\left(\partial^{\mu} U^{\dagger}\right)\left(\partial_{\mu} U\right), \quad U=e^{(i / F) \phi}, \quad \phi=\phi^{a} T^{a}$,

where $\mathrm{U} \in \mathrm{SU}(N)$ transforms under the general element $\left(V_{R}, V_{L}\right)$ of the chiral group $\mathrm{SU}(N) \times \mathrm{SU}(N)$ as

$$
U \rightarrow V_{R} U V_{L}^{-1},
$$

there are no cubic vertices, and the matrix $\phi$ of $N^{2}-1$ scalar fields transforms under the axial transformation $V_{L}=V_{R}^{-1}=1+i \alpha^{a} T^{a} \equiv 1+i \alpha$ (with $\alpha$ infinitesimal) as

$\delta_{\alpha} \phi=2 F \alpha-\frac{1}{6 F}\left\{\alpha, \phi^{2}\right\}+\frac{1}{3 F} \phi \alpha \phi+O\left(\phi^{3}, \alpha^{2}\right)$.

The linear term is absent, and consequently the theory has the Adler zero.

New soft theorem.-Let us assume a general twoderivative Lagrangian for $N$ fields $\left\{\phi_{I}\right\}_{I=1}^{N}$ with a cubic vertex,

$\mathcal{L}=\frac{1}{2} \partial_{\mu} \phi_{I} \partial^{\mu} \phi_{I}+\frac{1}{2} K_{I J K} \partial_{\mu} \phi_{I} \partial^{\mu} \phi_{J} \phi_{K}+\mathcal{O}\left(\phi^{4}\right)$,

with the sum over repeating indices tacitly assumed. Let the transformation of the fields corresponding to spontaneously broken symmetry contain, besides the constant term, also a linear term:

$$
\delta^{J} \phi_{I}=F_{I}^{J}+\sum_{K=1}^{N} C_{I K}^{J} \phi_{K}+\mathcal{O}\left(\phi^{2}\right) .
$$

The invariance of Eq. (12) under the symmetry (13) requires nontrivial constraints between all coefficients; namely, 


$$
B_{I K}^{J} \equiv C_{I K}^{J}+\frac{1}{2} \sum_{L=1}^{N} K_{I K L} F_{L}^{J}
$$

must be antisymmetric, $B_{I K}^{J}=-B_{K I}^{J}$. The Noether current $N_{\mu}^{J}$ contains a quadratic term in the field expansion,

$N_{\mu}^{J}=\sum_{I=1}^{N} F_{I}^{J} \partial_{\mu} \phi_{I}+\sum_{L, K=1}^{N} \mathcal{K}_{L K}^{J} \phi_{K} \partial_{\mu} \phi_{L}+\mathcal{O}\left(\phi^{3}\right)$

where $\mathcal{K}_{I K}^{J}$ depend on both parameters $C$ and $K$ :

$$
\mathcal{K}_{I K}^{J}=C_{I K}^{J}+\sum_{M=1}^{N} F_{M}^{J} K_{M I K}
$$

At the tree level the matrix element $\left\langle\alpha\left|N_{\mu}^{J}\right| \beta\right\rangle$ has additional singular terms from inserting the current into external legs. The remainder $R_{\mu}^{J}$ is not regular for $p \rightarrow 0$; hence, the soft limit of $p^{\mu} R_{\mu}^{J}$ is nonzero and reduces to

$$
\lim _{p \rightarrow 0} p^{\mu} R_{\mu}^{J}=-\sum_{L \in \alpha \cup \beta} \sum_{K=1}^{N} \mathcal{C}_{L K}^{J} A_{n-1}^{K, L}(\alpha, \beta),
$$

where the $A_{n-1}^{K, L}(\alpha, \beta)$ is the $(n-1)$ point amplitude and the particle $\phi_{L}\left(p_{L}\right)$ is omitted and is replaced by particle $\phi_{K}\left(p_{L}\right)$ with momentum $p_{L}$. The sum over $L$ is over the indices of all the particles in the in and out states. Therefore the soft theorem has the form

$\lim _{p \rightarrow 0} \sum_{I=1}^{N} F_{I}^{J} A_{n}\left(\alpha+\phi_{I}(p), \beta\right)=\sum_{I \in \alpha \cup \beta} \sum_{K=1}^{N} \mathcal{C}_{I K}^{J} A_{n-1}^{K, I}(\alpha, \beta)$.

Here the coefficient function $\mathcal{C}_{I K}^{J}$ is related to the original parameters in the Lagrangian and transformation as

$$
\mathcal{C}_{I K}^{J}=B_{I K}^{J}+\frac{1}{2} \sum_{M=1}^{N} F_{M}^{J}\left(K_{M I K}-K_{M K I}\right)=-\mathcal{C}_{K I}^{J} .
$$

However, since the on-shell amplitudes are invariant with respect to redefinition of the fields of the form $\phi_{I}=\phi_{I}^{\prime}+O\left(\phi^{\prime 2}\right)$, the constants $\mathcal{C}_{I K}^{J}$ do not depend on such a reparametrization of the Lagrangian. Note that several conditions must be satisfied in order to get a nonzero right-hand side of Eq. (18).

(1) The coefficients $\mathcal{C}_{I K}^{J}$ must be nonzero; i.e., no cancellation between parameters in Eqs. (12) and (13) occurs.

(2) The theory needs to have both even and odd amplitudes, as the amplitudes on the right-hand side have $(n-1)$ external legs. Most sigma models do have only even point amplitudes and, therefore, they preserve the Adler zero.
Example of the sigma model.-As an explicit example we consider a theory of two types of NGB fields: a vector of multiple complex scalar fields $\Phi_{I}^{+}, I=1, \ldots, N-1$, and a single real scalar $\chi$. We use the parametrization

$$
\hat{u}=\left(\begin{array}{c}
\frac{\Phi^{+}}{F} \\
\sqrt{1-\frac{\Phi^{-} \cdot \Phi^{+}}{F^{2}}}
\end{array}\right),
$$

where $\Phi^{+}=\left(\phi_{1}^{+}, \phi_{2}^{+}, \ldots, \phi_{N-1}^{+}\right)^{T}, \Phi^{-}=\left[\Phi^{+}\right]^{\dagger}$, and [.] stands for the contraction over the $I$ index. The Lagrangian of the model is

$$
\begin{aligned}
\mathcal{L}= & \frac{(\partial \chi)^{2}}{2}+F^{2}\left(\partial^{\mu} \hat{u}^{\dagger} \cdot \partial_{\mu} \hat{u}\right)+\frac{i F_{0}}{2} \partial^{\mu} \chi\left(\partial_{\mu} \hat{u}^{\dagger} \cdot u-\hat{u}^{\dagger} \cdot \partial_{\mu} \hat{u}\right) \\
& -\left(F^{2}-\frac{F_{0}^{2}}{2}\right)\left(\hat{u}^{\dagger} \cdot \partial^{\mu} \hat{u}\right)\left(\partial_{\mu} \hat{u}^{\dagger} \cdot \hat{u}\right) .
\end{aligned}
$$

It has two coupling constants $F, F_{0}$ which play the role of the decay constants of the NGB $\phi_{I}^{+}$and $\chi$, respectively. The model described by (21) is a different parametrization of the $\mathrm{SU}(N) / \mathrm{SU}(N-1)$ nonlinear sigma model (3). The relation with the original couplings is

$$
F_{0}=\frac{1}{\lambda}(1-\kappa)^{1 / 2}, \quad F=\frac{1}{\sqrt{2} \lambda} .
$$

Let us briefly summarize limiting cases of our model (for details and discussion, see Ref. [17]). The limit $\kappa \rightarrow 1$ gives $F_{0} \rightarrow 0$ and $\chi$ decouples: we get the $C P(N-1)$ model. The case $\lambda \rightarrow 0$ with $1-\kappa=O\left(\lambda^{2}\right)$ means $F \rightarrow \infty, F_{0}$ finite, and the theory is free. The limit $\kappa \rightarrow 0, \lambda$ fixed means $F_{0}=\sqrt{2} F$, which gives the $\mathrm{O}(2 N) / \mathrm{O}(2 N-1)$ model.

Note that the model (21) satisfies the first condition for the Adler zero violation as it involves the cubic term

$$
\mathcal{L} \ni i \frac{F_{0}}{2 F^{2}} \partial^{\mu} \chi\left(\partial_{\mu} \phi_{I}^{-} \cdot \phi_{I}^{+}-\partial_{\mu} \phi_{I}^{+} \cdot \phi_{I}^{-}\right) .
$$

The Lagrangian is derivatively coupled in the $\chi$ field, and it is therefore trivially invariant under the shift symmetry,

$$
\delta \chi=a .
$$

Since the cubic vertices can be eliminated by the reparametrization $\Phi^{ \pm}=\Phi^{ \pm \prime} \exp \left[ \pm i\left(F_{0} / 2 F\right) \chi\right]$, which does not spoil this property, all scattering amplitudes have the vanishing soft limit at $p_{\chi} \rightarrow 0$; i.e., for $\chi$ the Adler zero is valid. After this reparametrization, the Lagrangian is also invariant under a more complicated transformation involving the linear terms,

$$
\begin{aligned}
\delta \chi & =\frac{F_{0}}{2 F^{2}}\left(a_{I}^{-} \cdot \phi_{I}^{+}+a_{I}^{+} \cdot \phi_{I}^{-}\right)+\mathcal{O}\left(\left(\chi, \phi^{ \pm}\right)^{2}\right), \\
\delta \phi_{I}^{ \pm} & =\mp i a_{I}^{ \pm}\left(1 \mp \frac{F_{0}}{2 F^{2}} \chi\right)+\mathcal{O}\left(\left(\chi, \phi^{ \pm}\right)^{2}\right),
\end{aligned}
$$




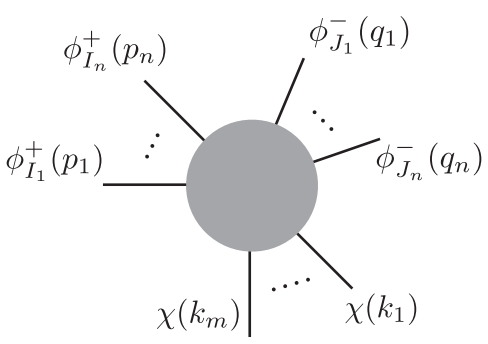

FIG. 1. Scattering amplitude $A_{M}\left(\left\{\phi_{I_{i}}^{+}\right\},\left\{\phi_{J_{j}}^{-}\right\},\{\chi\}\right)$.

where we introduced shift parameters $a_{I}^{ \pm}$. Note that the symmetry mixes the single scalar field $\chi$ and multiple scalars $\phi_{I}^{ \pm}$. Calculating $\mathcal{C}_{I K}^{J}$ in Eq. (19) we learn that $\mathcal{C}_{I K}^{J}$ is nonzero. Furthermore, the model involves both odd and even amplitudes, and, therefore, the scattering amplitude does not vanish when the momentum of one of $\phi_{I}^{ \pm}$is taken soft. Because of the form of the Lagrangian (21), the only allowed amplitudes have the same number of $\phi^{+}$and $\phi^{-}$ fields. If we think about $\phi^{ \pm}$as charged scalars, this just stands for charge conservation. Let us consider now the scattering amplitude of $2 n$ fields $\phi_{I}^{ \pm}$and $m$ fields $\chi$, with total $M=2 n+m$ external legs (see Fig. 1),

$$
\begin{aligned}
& A_{M}\left(\left\{\phi_{I_{i}}^{+}\right\},\left\{\phi_{J_{j}}^{-}\right\},\{\chi\}\right) \\
& \equiv A\left(\phi_{I_{1}}^{+}\left(p_{1}\right) \ldots \phi_{I_{n}}^{+}\left(p_{n}\right), \phi_{J_{1}}^{-}\left(q_{1}\right) \ldots \phi_{J_{n}}^{-}\left(q_{n}\right), \chi\left(k_{1}\right) \ldots \chi\left(k_{m}\right)\right) .
\end{aligned}
$$

The soft theorem when $p_{1} \rightarrow 0$ then reads

$$
\lim _{p_{1} \rightarrow 0} A_{M}=\frac{i F_{0}}{2 F^{2}} \sum_{i=1}^{m} A_{M-1}^{(i)}-\frac{i F_{0}}{2 F^{2}} \sum_{j=1}^{n} \delta_{I_{1} J_{j}} A_{M-1}^{(j)},
$$

where the lower point amplitudes are defined as follows:

$$
\begin{aligned}
& \left.A_{M-1}^{(i)} \equiv A\left(\phi_{I_{1}}^{+}\left(k_{i}\right) \ldots \phi_{I_{n}}^{+},\left\{\phi_{J_{j}}^{-}\right\}, \chi\left(k_{1}\right) \ldots \widehat{\chi\left(k_{i}\right)}\right) \ldots \chi\left(k_{m}\right)\right), \\
& \left.A_{M-1}^{(j)} \equiv A\left(\phi_{I_{2}}^{+} \ldots \phi_{I_{n}}^{+}, \phi_{J_{1}}^{-} \ldots \phi_{J_{j}}^{-\left(q_{j}\right.}\right) \ldots \phi_{J_{n}}^{-}, \chi\left(q_{j}\right),\{\chi\}\right) .
\end{aligned}
$$

In the first case, $A_{M-1}^{(i)}$, we start with $A_{M}$ defined in Eq. (26) and remove particle $\chi\left(k_{i}\right)$, then we replace the particle $\phi_{I_{1}}^{+}\left(p_{1}\right)$ by $\phi_{I_{1}}^{+}\left(k_{i}\right)$, i.e., just replace momenta keeping the quantum numbers the same, and finally sum over all particles $\chi\left(k_{i}\right)$ which are removed. In the case of $A_{M-1}^{(j)}$, we remove particle $\phi_{I_{1}}^{+}$completely as well as $\phi_{J_{j}}^{-}$, and add a new single scalar particle $\chi\left(q_{j}\right)$ with the momentum of removed $\phi^{-}$particle. We show this graphically in Fig. 2 (left-hand picture corresponds to $A^{(i)}$ while the right-hand side corresponds to $A^{(j)}$ ), where the red color stands for removed legs and blue for the legs added. For $q_{1} \rightarrow 0$, the soft theorem is the same except for the overall sign on the
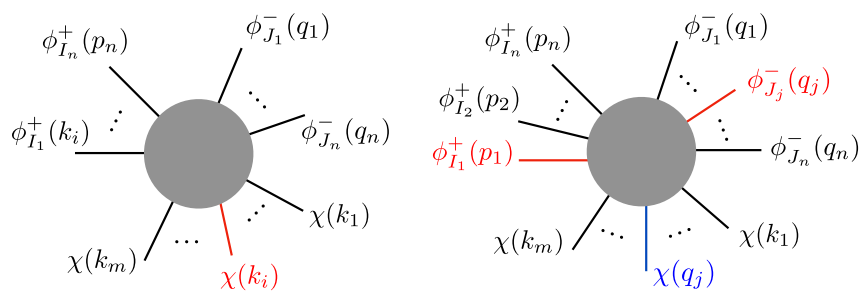

FIG. 2. Soft theorem for the amplitude $A_{M}$ involves two terms.

right-hand side of Eq. (27). As discussed earlier, any amplitude vanishes for $k_{j} \rightarrow 0$.

In the following, we focus now on the $N=2$ case which describes only three fields: $\phi^{ \pm}, \chi$. To check the soft theorem we first calculate all nonvanishing four-point amplitudes,

$$
\begin{aligned}
A_{4}^{\text {tree }}\left(\phi_{1}^{+}, \phi_{2}^{+}, \phi_{3}^{-}, \phi_{4}^{-}\right) & =\frac{1}{4 F^{4}}\left(3 F_{0}^{2}-8 F^{2}\right) s_{12}, \\
A_{4}^{\text {tree }}\left(\phi_{1}^{+}, \phi_{2}^{-}, \chi_{3}, \chi_{4}\right) & =\frac{F_{0}^{2}}{4 F^{4}} s_{12},
\end{aligned}
$$

where $s_{i j}=\left(p_{i}+p_{j}\right)^{2}$ and we used the notation $\phi_{1}^{+} \equiv$ $\phi^{+}\left(p_{1}\right)$ for simplicity. There is only one nontrivial fivepoint amplitude:

$A_{5}^{\text {tree }}\left(\phi_{1}^{+}, \phi_{2}^{+}, \phi_{3}^{-}, \phi_{4}^{-}, \chi_{5}\right)=\frac{i F_{0}}{F^{6}}\left(F^{2}-\frac{F_{0}^{2}}{2}\right)\left(s_{12}-s_{34}\right)$.

The soft theorem (27) for $p_{1} \rightarrow 0$ predicts

$$
\begin{aligned}
\lim _{p_{1} \rightarrow 0} A_{5}^{\text {tree }}= & \frac{i F_{0}}{2 F^{2}} A_{4}^{\text {tree }}\left(\phi_{5}^{+}, \phi_{2}^{+}, \phi_{3}^{-}, \phi_{4}^{-}\right) \\
& -\frac{i F_{0}}{2 F^{2}}\left[A_{4}^{\text {tree }}\left(\phi_{2}^{+}, \phi_{3}^{-}, \chi_{4}, \chi_{5}\right)+A_{4}^{\text {tree }}\left(\phi_{2}^{+}, \phi_{4}^{-}, \chi_{3}, \chi_{5}\right)\right] \\
= & -\frac{i F_{0}}{F^{6}}\left(F^{2}-\frac{F_{0}^{2}}{2}\right) s_{34}
\end{aligned}
$$

in agreement with the direct calculation (29).

Amplitude reconstruction.-The knowledge of the soft theorem (27) can be used as an input in the modified version of the soft recursion relations introduced in Ref. [8]. We start with the momentum shift where all but two particles are shifted in the way that allows us to access the soft limit,

$$
\begin{aligned}
& \hat{p}_{i}=\left(1-a_{i} z\right) p_{i}, \quad i=1, \ldots n-2, \\
& \hat{p}_{j}=p_{j}+z q_{j}, \quad j=n-1, n,
\end{aligned}
$$

where the parameters $a_{i}$ and vectors $q_{j}$ must preserve onshell conditions and momentum conservation. For this shift any scattering amplitude scales like $A_{n}^{\text {tree }}(z)=\mathcal{O}\left(z^{2}\right)$, based on the momentum counting. We consider a residue theorem for the meromorphic function $G_{n}(z)$ : 


$$
G_{n}(z) \equiv \frac{A_{n}^{\text {tree }}(z)}{z \prod_{i}\left(1-a_{i} z\right)} .
$$

We need at least three factors of $\left(1-a_{i} z\right)$ in the denominator to have vanishing residue at $z \rightarrow \infty$; i.e.,

$$
\lim _{R \rightarrow \infty} \oint_{|z|=R} d z G_{n}(z)=0
$$

We can then express the residue at $z=0$, the original amplitude $A_{n}^{\text {tree }}$, as the sum of all other residues:

$$
A_{n}^{\text {tree }}=-\sum_{k} \operatorname{Res}_{z=z_{k}} G_{n}(z)-\sum_{i} \operatorname{Res}_{z=1 / a_{i}} G_{n}(z) .
$$

The first sum on the right-hand side refers to factorization poles from $A_{n}(z)$; each term is equal to the product of corresponding lower point amplitudes. The second sum is over the soft limit poles when one of the $\hat{p}_{j} \rightarrow 0$. In Ref. [8] we considered only theories with vanishing soft limits, i.e., the second sum never contributed, but now the contribution is nonzero and it is given by Eq. (18). The same concept was also studied in Ref. [18].

As an example, we will reconstruct the five-point amplitude from the $N=2$ model, $A_{5}^{\text {tree }}\left(\phi_{1}^{+}, \phi_{2}^{+}, \phi_{3}^{-}, \phi_{4}^{-}, \chi\right)$. We shift legs 1, 2, and 5 as Eq. (31) and 3 and 4 as Eq. (32). The amplitude does not have any factorization poles, and the only poles of $G_{5}(z)$ are soft poles. As the shifted amplitude vanishes for $\hat{p}_{5} \rightarrow 0$, the only contributions come from $\hat{p_{1}}$ or $\hat{p_{2}} \rightarrow 0$ soft limits. The residue at $z=1 / a_{1}$ then reads

$$
\operatorname{Res}_{z=1 / a_{1}} G_{5}(z)=-\frac{\left.\widehat{A_{5}}\right|_{z=1 / a_{1}} ^{\text {tree }}}{\left(1-a_{2} / a_{1}\right)\left(1-a_{5} / a_{1}\right)} .
$$

The value of the shifted amplitude $\left.\widehat{A_{5}}\right|_{z=1 / a_{1}} ^{\text {tree }}$ can be obtained from the soft theorem (30) by considering the shifted kinematics:

$$
\hat{p_{1}}=0, \quad \hat{p_{2}}=\left(\frac{a_{1}-a_{2}}{a_{1}}\right) p_{2}, \quad \hat{p_{5}}=\left(\frac{a_{1}-a_{5}}{a_{1}}\right) p_{5} .
$$

Plugging the result into Eq. (36), we get

$$
\operatorname{Res}_{z=1 / a_{1}} G_{5}(z)=\frac{i F_{0}}{F^{6}}\left(F^{2}-\frac{F_{0}^{2}}{2}\right) s_{25} .
$$

Similarly, the residue at the pole $z=1 / a_{2}$ for $\hat{p_{2}}=0$ gives

$$
\operatorname{Res}_{z=1 / a_{2}} G_{5}(z)=\frac{i F_{0}}{F^{6}}\left(F^{2}-\frac{F_{0}^{2}}{2}\right) s_{15},
$$

and after using the momentum conservation the sum of Eqs. (37) and (38) reproduces the formula (29).
Uniqueness of the model.--In the last part we turn the procedure around, and we will reconstruct our nonlinear sigma model for $N=2$ as a unique theory which satisfies a soft theorem of the type Eq. (18). Following the logic of Ref. [9], we start with the ansatz for the amplitude of three types of scalar fields $\phi^{ \pm}, \chi$ in terms of kinematical invariants and impose the soft theorem of the general type,

$$
\lim _{p_{1} \rightarrow 0} A_{n}=\sum_{i} c_{i} A_{n-1}^{(i)},
$$

as a constraint. If the right-hand side is zero, we deal with the standard Adler theorem (for more details, see Ref. [10]). To go beyond the standard situation we demand a nonzero right-hand side when shifting charged particles, and keep the Adler zero only for the neutral $\chi$. We went up to the seven-point amplitudes to check that the unique answer is our model, $\mathrm{U}(1)$-fibrated $\mathrm{CP}(1)$, and the general $c_{i}$ constants are set in accordance with Eq. (27).

The natural question is if there are more theories of this type for more than three scalar fields beyond our explicit example (21). This is an open question, and we believe that this procedure is a very useful tool to address the problem and potentially find new theories with nontrivial soft theorems. In principle, we can also look at amplitudes for theories with only two types of scalar fields. In the upcoming work [17] we will prove that for any such theory, under the assumption that the soft theorem (18) with $F_{I}^{J}=$ $F \delta_{I}^{J} \neq 0$ is valid, and assuming nonvanishing four-point amplitude, all the odd-particle amplitudes have to vanish. Therefore all Goldstone-boson amplitudes must necessarily have the Adler zero. This supports the statement that the only nonlinear sigma model for two scalars are $\mathrm{CP}(1)=$ $\mathrm{O}(3) / \mathrm{O}(2)$ and $\mathrm{O}(1,2) / \mathrm{O}(2)$.

Conclusion.-In this Letter we found a new soft theorem for the Goldstone-boson amplitudes. Using the example of $\mathrm{SU}(N) / \mathrm{SU}(N-1)$ nonlinear sigma models, we showed that generically the amplitudes do not vanish in the soft limit but rather reduce to a recursion. Explicit expressions are presented in the simplest $N=2$ case which describes a pair of charged NGBs and a single neutral NGB. We proved that this theory can be uniquely fixed from the tree-level $S$ matrix if we impose the soft theorem as a constraint. Consequently, we derived the recursion relations to reconstruct all tree-level amplitudes.

Our work opens new avenues in studying NLSMs, and more generally EFTs using nonvanishing soft limits of scattering amplitudes. In Ref. [17] we will generalize this work, and use the soft theorems as the theoretical tool to explore larger space of theories based on properties of their scattering amplitudes. The exceptional EFTs also appear in the Cachazo-He-Yuan formula [19], ambitwistor strings [20], and the color-kinematics duality [21], while the nontrivial soft limits have been encountered in the calculation of the leading nonzero term in the soft limit of SU(N) 
NLSM amplitudes using the Cachazo-He-Yuan formalism [22]. In Ref. [23] the authors studied the amplitudes of dilaton, and later in Refs. [24-26] the subleading soft theorem for such amplitudes was derived. The soft theorem constraints on EFT of $N=4$ Super Yang-Mills theory (SYM) on the Coulomb branch for both dilaton and the Goldstone of $R$-symmetry breaking was studied in Ref. [27], and the nonvanishing soft theorem of string dilation was found in Ref. [28]. It would be fascinating to explore how these results can be understood using our approach.

This work is supported in part by the Czech Government projects GACR 18-17224S and LTAUSA17069, by U.S. DOE Grants No. DE-SC0009999 and No. DE-SC0011842, and the funds of University of California.

[1] C. D. Batista, M. Shifman, Z. Wang, and S. S. Zhang, Phys. Rev. Lett. 121, 227201 (2018).

[2] P. Azaria, B. Delamotte, and T. Jolicoeur, Phys. Rev. Lett. 64, 3175 (1990); P. Azaria, B. Delamotte, F. Delduc, and T. Jolicoeur, Nucl. Phys. B408, 485 (1993).

[3] D. Schubring and M. Shifman, Phys. Rev. D 101, 045003 (2020).

[4] A. B. Zamolodchikov and A. B. Zamolodchikov, Ann. Phys. (N.Y.) 120, 253 (1979).

[5] A. M. Polyakov and P. B. Wiegmann, Phys. Lett. 131B, 121 (1983).

[6] P. B. Wiegmann, Phys. Lett. 141B, 217 (1984); 142B, 173 (1984); 152B, 209 (1985).

[7] R. Britto, F. Cachazo, and B. Feng, Nucl. Phys. B715, 499 (2005); R. Britto, F. Cachazo, B. Feng, and E. Witten, Phys. Rev. Lett. 94, 181602 (2005); T. Cohen, H. Elvang, and M. Kiermaier, J. High Energy Phys. 04 (2011) 053; C. Cheung, C. H. Shen, and J. Trnka, J. High Energy Phys. 06 (2015) 118.

[8] C. Cheung, K. Kampf, J. Novotny, C. H. Shen, and J. Trnka, Phys. Rev. Lett. 116, 041601 (2016).

[9] C. Cheung, K. Kampf, J. Novotny, and J. Trnka, Phys. Rev. Lett. 114, 221602 (2015).
[10] C. Cheung, K. Kampf, J. Novotny, C. H. Shen, and J. Trnka, J. High Energy Phys. 02 (2017) 020; J. Bijnens and K. Kampf (to be published).

[11] C. Cheung, K. Kampf, J. Novotny, C. H. Shen, J. Trnka, and C. Wen, Phys. Rev. Lett. 120, 261602 (2018).

[12] H. Elvang, M. Hadjiantonis, C. R. T. Jones, and S. Paranjape, J. High Energy Phys. 01 (2019) 195.

[13] I. Low and Z. Yin, J. High Energy Phys. 11 (2019) 078.

[14] N. Arkani-Hamed, L. Rodina, and J. Trnka, Phys. Rev. Lett. 120, 231602 (2018); L. Rodina, Phys. Rev. Lett. 122, 071601 (2019); L. Rodina, J. High Energy Phys. 09 (2019) 084; J. J. M. Carrasco and L. Rodina, Phys. Rev. D 100, 125007 (2019).

[15] L. Susskind and G. Frye, Phys. Rev. D 1, 1682 (1970).

[16] K. Kampf, J. Novotny, and J. Trnka, Phys. Rev. D 87, 081701(R) (2013); K. Kampf, J. Novotny, and J. Trnka, J. High Energy Phys. 05 (2013) 032; I. Low and Z. Yin, Phys. Rev. Lett. 120, 061601 (2018); J. Bijnens, K. Kampf, and M. Sjö, J. High Energy Phys. 11 (2019) 074.

[17] K. Kampf, J. Novotny, M. Shifman, and J. Trnka (to be published).

[18] H. Luo and C. Wen, J. High Energy Phys. 03 (2016) 088.

[19] F. Cachazo, S. He, and E. Y. Yuan, Phys. Rev. Lett. 113, 171601 (2014); J. High Energy Phys. 07 (2015) 149.

[20] E. Casali, Y. Geyer, L. Mason, R. Monteiro, and K. A. Roehrig, J. High Energy Phys. 11 (2015) 038.

[21] C. Cheung, C. H. Shen, and C. Wen, J. High Energy Phys. 02 (2018) 095; Z. Bern, J. J. Carrasco, M. Chiodaroli, H. Johansson, and R. Roiban, arXiv:1909.01358.

[22] F. Cachazo, P. Cha, and S. Mizera, J. High Energy Phys. 06 (2016) 170.

[23] Y.t. Huang and C. Wen, J. High Energy Phys. 12 (2015) 143.

[24] M. Ademollo, A. D’Adda, R. D'Auria, F. Gliozzi, E. Napolitano, S. Sciuto, and P. Di Vecchia, Nucl. Phys. B94, 221 (1975).

[25] J. A. Shapiro, Phys. Rev. D 11, 2937 (1975).

[26] P. Di Vecchia, R. Marotta, M. Mojaza, and J. Nohle, Phys. Rev. D 93, 085015 (2016).

[27] M. Bianchi, A. L. Guerrieri, Y.t. Huang, C. J. Lee, and C. Wen, J. High Energy Phys. 10 (2016) 036.

[28] Y. Wang and X. Yin, arXiv:1505.05861; M. B. Green and C. Wen, J. High Energy Phys. 06 (2019) 087. 